\title{
Le « carnet de santé 1996 » : autopsie d'un support de communication mort-né
}

The 1996 "Health Logbook": Autopsy of a Communication Medium Born Dead

\section{Olivier Chantraine}

\section{(2) OpenEdition}

\section{Journals}

Édition électronique

URL : http://journals.openedition.org/edc/1138

DOI : $10.4000 /$ edc. 1138

ISSN : 2101-0366

Éditeur

Université Lille-3

Édition imprimée

Date de publication : 1 mars 2001

Pagination : $51-68$

ISBN : 2-9514961-1-7

ISSN : $1270-6841$

Référence électronique

Olivier Chantraine, "Le "carnet de santé 1996 » : autopsie d'un support de communication mort-né », Études de communication [En ligne], 23 | 2001, mis en ligne le 10 janvier 2010, consulté le 03 mai 2019. URL : http://journals.openedition.org/edc/1138; DOI : 10.4000/edc. 1138

Ce document a été généré automatiquement le 3 mai 2019.

(c) Tous droits réservés 


\title{
Le « carnet de santé 1996 »: autopsie d'un support de communication mort-né
}

The 1996 "Health Logbook": Autopsy of a Communication Medium Born Dead

\author{
Olivier Chantraine
}

1 Au troisième semestre 1996, en France, la question de la santé publique est au cœur des conflits politiques et sociaux. En particulier un topique récurrent fait régulièrement le sujet des débats, des éditoriaux des presses écrite, radiophonique et audiovisuelle : le «trou de la sécu »1. C'est-à-dire le déficit des comptes des organismes de sécurité sociale. Une question politique conflictuelle oppose deux conceptions du financement et de la gestion de cette sécurité sociale :

- l'une fondée sur la cogestion par les «partenaires sociaux» - i.e. les syndicats patronaux et de salariés - avec pour références d'une part les équilibres de pouvoir nés à la Libération de la France en 1945 et d'autre part le mode de financement via un double prélévement sur les rémunérations salariales : cotisation employeur d'une part, cotisation salarié d'autre part,

- l'autre fondée sur une gestion par l'État de la sécurité sociale avec une progressive fiscalisation de son financement, déjà initiée à cette date par une «Contribution Sociale Généralisée ».

2 C'est dans ce contexte que le Ministère du Travail et des Affaires Sociales et le Secrétariat d'État à la Santé et à la Sécurité Sociale prennent l'initiative de créer un nouvel outil de communication et de gestion supposé permettre une nouvelle coordination du travail des divers professionnels de santé entre eux, avec les organismes de gestion et de contrôle et aussi avec un usager réputé «nomade»: le «nomadisme médical», c'est-à-dire la consultation de multiples médecins généralistes et spécialistes à l'initiative du seul patient, étant dans ce contexte considéré comme une difficulté majeure. Ce «nomadisme médical » est devenu par ailleurs le nom par lequel se désignent de manière récurrente dans l'environnement médiatique à la fois la source officiellement principale des maux du système de sécurité sociale et un prétendu problème de santé publique. En effet cette néfaste errance des patients empêche la coordination et la mémoire des soins par un 
nouveau personnage que les pouvoirs publics veulent voir au centre du dispositif de soins : le « médecin référent ».

D'autres acteurs sont évidemment à prendre en compte, tels l'industrie pharmaceutique, une myriade d'associations concernées par diverses questions de santé et de prévention, les diverses mutuelles, les syndicats et organismes professionnels. Le problème enfin n'est pas strictement national dans la mesure où la question des vaccinations est inséparable de celle des passages de frontières.

Cet outil, c'est le « carnet de santé » institué en 1996, qui pourrait être considéré comme la tentative de généraliser à l'ensemble de la population le carnet de santé concernant les mineurs, inséparable depuis plusieurs décennies de la politique de l'enfance.

Ce nouveau support a connu très peu de succès. L'essentiel des partenaires supposés en faire usage l'ont négligé, beaucoup sans même s'en apercevoir mais beaucoup aussi délibérément. D'autres dispositifs organisés autour de cartes électroniques et de réseaux de communication électronique - «Vitale 1 », puis « Vitale 2 »- ont ensuite pris le relais. L'analyse de ces cartes et réseaux électroniques ne sera pas entreprise dans le présent papier.

6 On proposera ici une description sociosémiotique de ce "carnet de santé », considéré comme un support et un outil de communication. Cette description prendra appui sur l'hypothèse, formulée par ailleurs (Chantraine, 1997) qu'une forme sociosémiotique, «l'espace public professionnel », structure les représentations et les relations des acteurs inscrits professionnellement dans des dispositifs publics d'action.

7 Après le rappel de quelques aspects essentiels de cette forme, on s'attachera à montrer comment le «carnet de santé 1996 » apparait comme une tentative volontariste d'imposer un nouvel espace public professionnel par voie autoritaire, au mépris de l'incontournable processus complexe de construction et de négociation sociale continue d'un tel espace. En conclusion on proposera d'évaluer la démarche qui consiste à s'en remettre à des outils et supports de communication pour modifier des espaces de relations sociales. Paraphrasons un adage devenu célèbre: change-t-on un espace professionnel en instaurant par décret l'obligation d'usage d'un nouveau support de communication?

\section{"L'espace public professionnel » : une forme sociosémiotique qui structure et unifie un réseau de signifiants de diverses substances et natures}

8 On a proposé d'appeler « espace public professionnel » (Chantraine, 1997) la forme dans laquelle un acteur impliqué dans un dispositif public d'action inscrit sa pratique. On en rappelera ici les traits principaux en les illustrant par leur mode d'existence dans le champ médical, de manière à pouvoir ensuite confronter ces traits à la tentative de les modifier qu'a été l'instauration du « carnet de santé ».

Cette forme structure et unifie notamment : 


\section{l'espace graphique de la page d'écriture}

10 C'est un aspect bien connu de la pratique quotidienne de la médecine que la réalisation de tout une série de pages d'écriture normées par diverses conventions et réglementations : ordonnances de diverses catégories selon le statut réglementaire des produits ou prestations prescrits, feuilles de sécurité sociale, arrêts de travail, courriers confraternels, pages diversement organisées de divers formulaires etc... Selon les cas, le médecin a fait réaliser lui-même les supports - papiers à en-tête de diverses présentations - et y garde un espace relativement libre pour la mise en espace de son écriture et de sa signature ou au contraire il doit utiliser le formatage défini auparavant par diverses institutions. Dans ce dernier cas, il se plie plus ou moins aux cadres qui lui sont impartis.

De même il peut parfois écrire selon la norme commune du langage mais souvent aussi il utilisera un vocabulaire savant ou technique, voire des codes accessibles seulement aux professionnels de la santé et aux organismes de gestion.

L'usage de tous ces formats et le respect de toutes ces normes par le médecin sont à la fois le symptôme de ses relations avec les patients, les institutions, et les autres professionnels et un aspect de la reproduction quotidienne de ces relations.

\section{l'espace proxémique de la pratique professionnelle quotidienne}

Les lieux de la pratique médicale sont organisés par la répartition de divers espaces où les conventions de communication sont différentes.

14 Ainsi dans le cas de la consultation d'un «médecin de ville», l'accueil est assuré généralement par un secrétariat anonyme qui manipule agenda et téléphone et oriente le patient vers une salle d'attente.

Dans celle-ci, le patient peut entretenir une conversation avec d'autres patients, lire des journaux et magazines d'actualité plus ou moins récente, s'éduquer par la lecture d'affiches relatives à l'alcoolisme, la prévention de diverses maladies, la vaccination. Souvent même il sera la cible de messages publicitaires, soit relatifs à des prestations médicales spécifiques, telles que le traitement de la calvitie, soit liés à toutes sortes d'annonceurs pour lesquels a été réalisé un programme vidéo spécifique diffusé en boucle sur l'écran d'un moniteur. Toutes ces communications ont vocation à être interrompues immédiatement sitôt que le médecin intervient.

Reçu enfin par le médecin, le patient passe ensuite par diverses configurations spatiales selon la spécialité du médecin : poignée de mains souvent dans l'embrasure de la porte de la salle d'attente, sous le regard des autres patients, puis entretien à huis clos, assis face à face de chaque côté d'un bureau, sur lequel trône maintenant le plus souvent un ordinateur.

Le téléphone permet l'interruption de ce dialogue pour le médecin mais, bien évidemment, pas pour le patient.

Parfois ensuite se produit le passage en salle technique où les règles de la pudeur et de la distance entre les corps changent. Souvent des dispositifs spécifiques d'éclairage artificiel organisent cet espace d'examen. Un(e) assistant(e) est susceptible d'y être appelé(e). De même le patient peut être amené à s'y mouvoir non pas de lui-même mais par l'effet de 
divers appareils mécaniques. Sa parole enfin n'y a pas le même statut que lors du premier entretien.

L'examen ou les soins prenant fin, le rituel de clôture se déroule à nouveau dans le bureau du médecin, généralement debout, parfois ponctué par la poignée de mains et l'échange de propos ordinaires selon le degré de familiarité possible entre le client et le médecin, selon leurs affinités culturelles et l'ancienneté de leur relation. Une nouvelle interaction avec l'accueil n'est pas toujours indispensable.

Chacun sait bien qu'il n'en va pas de même dans une clinique radiologique, un service d'urgence, une consultation dans un dispensaire, une chambre d'hôpital. On perçoit bien pourtant que tous ces lieux sont des espaces structurés par des règles et des routines complexes qui sont à la fois le reflet et un mode de reproduction des relations légitimes qui supportent les interactions. Chacun se coordonne selon son statut et les conventions proxémiques inséparables des lieux et des pratiques qui s'y inscrivent.

\section{l'espace géopolitique de l'action publique}

On a vu ci-dessus que l'espace de l'interaction à huis clos était relié à l'espace extérieur par le téléphone et souvent par l'ordinateur. C'est sous cet aspect technologique que les liens entre l'espace graphique et l'espace proxémique d'une part et l'espace géopolitique de l'action publique d'autre part sont les plus visibles. Pourtant l'ordonnance présuppose elle aussi les relations entre le prescripteur, le visiteur médical, le laboratoire pharmaceutique, l'officine où le patient va se faire délivrer ses produits médicamenteux et parfois l'infirmière qui se chargera de les lui administrer. Surtout cette ordonnance est accompagnée de la feuille de soins destinée à la Sécurité sociale, feuille qui inscrit cet ensemble de relations et de pratiques dans l'action publique, administrée et gérée par les organismes compétents. Autour de l'acte médical est tissé tout un réseau institutionnel, professionnel, économique, administratif, juridique et relationnel. Sa cartographie renvoie à la structuration du territoire de la santé publique, dans lequel la pratique médicale trouve son sens géopolitique.

\section{Approche textuelle du carnet de santé}

Le carnet de santé a été adressé de diverses manières aux assurés sociaux. Dans la mesure où un certain nombre de mutuelles lui avaient acordé leur soutien, il a été envoyé à leurs adhérents sous enveloppe transparente, avec un étrange courrier d'accompagnement qui tentait de poser un système de relations et de conventions entre usagers, professionnels et autorités. Ce courrier était "signé » par deux ministres et insistait sur le caractère « personnel » du carnet.

Le carnet lui-même se présentait d'une part comme un objet hybride, difficile à classer dans un genre communicationnel identifiable, et d'autre part un texte paradoxal dans sa disposition.

On proposera donc maintenant d'analyser :

a) le dispositif énonciatif du courrier d'accompagnement

b) la spécificité générique du carnet de santé

c) les paradoxes de la disposition générale du document. 
On verra que chacun de ces aspects renvoie à la tentative volontariste de modifier un espace public professionnel par l'assignation autoritaire aux acteurs qui y évoluent de rôles, droits et devoirs dans un processus d'écriture.

\section{A - Le dispositif énonciatif du courrier d'accompagnement}

Pour ce qui concerne les adhérents à des mutuelles, le carnet de santé leur a été envoyé par celles-ci. De sorte que l'analyse de ce courrier nécessite de faire une distinction entre :

- l'expéditeur, au sens postal du terme, d'une part, à savoir la mutuelle

- et le destinateur d'autre part, au sens sémiotique du terme, - celui qui annonce une intention de communication -, à savoir les deux ministres signataires du courrier d'accompagnement : Jacques Barrot, ministre du travail et des affaires sociales et Hervé Gaymard, secrétaire d'état à la santé et à la sécurité sociale.

En quelque sorte les mutuelles se sont faites les vaguemestres des autorités ministérielles qui, elles, avaient un «message » à transmettre, du sens à faire passer et se sont servi d'un dispositif communicationnel, le "courrier aux adhérents", dans lequel les sociétaires ont l'habitude de se reconnaître, et de se sentir en sécurité.

C'est ce qu'expriment bien les premières phrases de la lettre :

«Madame, Mademoiselle, Monsieur,

Ceci est votre carnet de santé.

Il a été conçu par le Ministère chargé de la santé, approuvé par l'Ordre des médecins et diffusé par votre organisme d'assurance maladie $»^{2}$.

Nous avons donc :

- un destinateur, l'État,

- une co-autorité, l'Ordre des médecins

- et un organisme technique de diffusion, l'organisme d'assurance maladie.

Il s'agit d'une configuration médiatique originale : l'État s'adresse aux citoyens via les moyens techniques de communication d'une organisation ne relevant pas principalement ni à première vue du secteur médiatique, ni de l'industrie de la communication. Cette organisation et l'Ordre des médecins laissent les ministres décrire leurs position et action et ne disent rien directement...

Autre paradoxe : la lettre est ponctuée de mots et de syntagmes en caractères gras qui tendent à attribuer au carnet de santé un certain nombre de qualités qui sont précisément celles qui lui manquent :

«Personnel, confidentiel, obligatoire, votre carnet de santé est la mémoire de votre santé».

Plus loin le terme « confidentialité » est répété, dans le contexte proche de «liberté».

Chacun de ces mots est une dénégation, en ceci qu'il renvoie précisément chaque fois à une carence communicationnelle du support :

- «Personnel»? : le « carnet de santé » est adressé à « Madame, Mademoiselle, Monsieur »... L'application de la règle de l'anonymat impose que la personne n'y laisse aucune trace, elle est donc uniquement identifiable et gérable via le numéro de sécurité sociale. Ainsi le carnet de santé ne connait rien de la personne, il gère et piste l'individu via le numéro de sécurité sociale (numéro qui à la même époque est devenu légalement accessible aux services fiscaux...). Enfin le carnet porte, en marge de son dos, les références de l'imprimerie nationale : 
« UCANSS - S3325A - IMPRIMERIE NATIONALE 09-96 ». voulu traçable.

- "Obligatoire » ? : en fait l'immense majorité des carnets sont restés inutilisés... et on ne s'en est guère inquiété. Nous sommes devant un consensus d'ordre cognitif d'une part, stratégique d'autre part:

- cognitif : les acteurs s'accordant tacitement pour savoir que les autorités resteraient là sans force,

- stratégique : les acteurs se coordonnant tacitement dans une attitude évidente de résistance, par référence à des contrats communicationnels antérieurs que cette résistance a contribué à refonder.

En tout cas ils savaient qu'ils ne seraient «obligés » à rien et se sont comportés de manière à ce que les faits leur donnent raison.

- "Votre carnet de santé est la mémoire de votre santé»: cette phrase est curieuse: elle décrit l'avenir mais est conjuguée à l'indicatif présent. Aussi cette étrange prophétie est-elle démentie d'entrée de jeu : le document est vierge au moment même où il est décrit comme porteur d'une mémoire...

Apparentée au slogan publicitaire ou de propagande, qui cherche vainement à faire exister du réel par des mots, elle est prolongée par une description à l'indicatif présent elle aussi :

«Il vous permet d'être mieux soigné. Il favorise la prévention. Grâce au carnet, votre médecin peut mieux vous suivre. C'est un nouveau trait d'union avec votre médecin, dans la totale liberté de choix du praticien et la stricte confidentialité du secret médical ».

41 La lecture de ces propos optimistes produit un effet d'ironie dans la mesure où les faits visibles au même moment les contredisent. Le discours des autorités, s'imposant dans un contexte de communication où il n'a pas sa place, apparait comme un discours de propagande sans prise sur un réel qui lui résiste ironiquement, invitant, en fait, et malgré lui, les acteurs à se coordonner tacitement pour la résistance passive.

\section{B - La spécificité générique du carnet de santé}

42 Après son courrier d'accompagnement, lisons maintenant le carnet de santé lui-même. Il apparait comme un objet textuel hybride, de genre mal déterminé et dont les règles incertaines assurent mal le succès.

43 On l'a vu plus haut, le carnet ressort de la catégorie des documents administratifs : il est référencé à l'imprimerie nationale et estampillé « Ministère chargé de la santé ». 


$$
\begin{aligned}
& \text { « } 6 \text { - SUIVI MEDICAL } \\
& 8 \text { - Renseignements médicaux importants } \\
& 14 \text { - Consultations et examens } \\
& 36 \text { - Hospitalisations } \\
& 40 \text { - Radiologie et imagerie médicale } \\
& 44 \text { - Vaccinations". }
\end{aligned}
$$

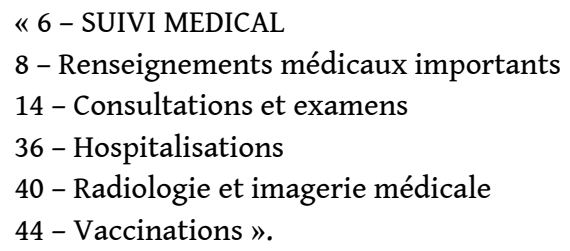

classique : l'organisation spatiale et temporelle du discours. Nous nous attacherons à la partie principale du document, soit les pages 6 à 47, regroupées sous un sur-titre : «suivi médical ». La disposition de cette partie du document est présentée par un sommaire :

51 A titre expérimental, ce sommaire a été soumis à trois groupes d'étudiants, aucun n'est parvenu à identifier pourquoi les vaccinations ne semblent pas pouvoir s'effectuer dans le cadre de consultations, pourquoi on ne fait ni radio ni imagerie à l'hôpital, pourquoi les renseignements médicaux importants ne sont pas liés aux examens, pourquoi on ne pratique pas d'examens à l'hôpital etc... 

le compte-rendu du «suivi médical»? C'est la comparaison des diverses pages préformatées de chacune des parties qui peut nous éclairer sur ce point. Ces pages renvoient chacune à des espaces proxémiques spécifiques caractéristiques des grands types d'exercice de la médecine et des relations professionnels-clients - comme nous les avons approchés plus haut de manière rapidement descriptive. Dans chacune de ces pages, le format est révélateur du type de relation, des règles administratives, économiques et déontologiques qui s'y appliquent. Les mentions paratextuelles tendent à donner à l'usager un mode d'emploi des lieux, à définir les règles de comportement applicables aux uns et aux autres. Souvent ce mode d'emploi et ces règles sont en contradiction avec les règles de communication admises par les acteurs concernés de cet espace public professionnel. On verra ainsi que l'ensemble du document, qui a vocation à «suivre » - tracer? - les pérégrinations de notre nomade médicalisé tend à gérer les trajets entre les lieux et à définir la pratique légitime dans chacun des lieux.

Ces lieux sont principalement :

- le cabinet du médecin « de ville $»^{3}$, où il est prévu de remplir les rubriques « renseignements médicaux importants » et «Consultations et examens ». Ce lieu se voit attribuer les pages 8 à 35 , soit plus de la moitié du document. Il peut aussi être concerné par la rubrique «Vaccinations" qui occupe les pages 40 à 47 , mais où la pratique est formalisée très différemment comme nous le montrerons ci-dessous ${ }^{4}$.

- l'hôpital ou la clinique, où il est prévu de remplir la rubrique "hospitalisations", pour laquelle sont prévues les pages 36 à 39.

- le cabinet de radiologie ou d'imagerie, pour la rubrique « radiologie et imagerie médicale » s'est vu attribué les pas 40 à 43.

Les principales différences entre ces espaces graphiques renvoient directement aux règles de la communication sur les lieux auxquels ils font référence :

\section{dans le cabinet de ville,}

le médecin est invité à écrire sous les rubriques suivantes :

«Motifs de la consultation et constatations utiles

Actes et prescriptions éventuels

Date cachet et signature du médecin ».

Ainsi les rôles sont bien répartis : d'un côté le patient est « motivé », de l'autre le médecin " constate » des faits et trie ce qui est «utile », pertinent. Il est prudemment rappelé au médecin qu'il n'est pas toujours nécessaire de "prescrire » ou "d'agir ». Enfin la coprésence du patient et du médecin est requise : datée, cachetée et surtout signée, la signature étant la preuve de la présence physique du médecin.

Dans le cas des "Vaccinations », le format textuel est très différent. Les rubriques sont :

«Vaccin (Diphtérie, Tétanos, Polio, Hépatite B, Rubéole, autre vaccin, BCG)

Date de la dernière vaccination ou du dernier rappel

Marque et lot

Date cachet et signature du médecin »

(Pour le BCG deux rubriques supplémentaires) : «date du dernier contrôle » et « résultat du contrôle ».

Ainsi dans le même lieu et la même présence étant requise, le médecin n'a pas du tout la même tâche d'écriture quand il vaccine ou quand il consulte simplement, agit d'une autre manière ou prescrit autre chose qu'un vaccin. Cette partie du document s'apparente au 
"carnet de vaccination " naguère sésame indispensable pour l'inscription des enfants dans les écoles, utile pour le passage de certaines frontières. La signature du médecin ne suffit pas pour prouver la réalité de la vaccination : sa démarche intellectuelle - calcul des dates de rappel, contrôle du BCG - doit aussi être traçable ainsi que le produit fourni par l'industrie pharmaceutique. De même que le certificat de vaccination antirabique des chiens et des chats, cette partie du document renvoie à l'espace de la santé publique dans tous ses aspects, y compris l'aspect international. Il positionne par ailleurs le médecin comme le dernier maillon de la chaîne de l'industrie pharmaceutique : il a la charge de la traçabilité du produit.

\section{A l'hôpital ou à la clinique,}

«A votre entrée, une charte du patient hospitalisé vous sera remise, avec un livret

d'accueil. Cette charte vous permettra de connaître vos droits à l'hôpital ».

\section{Au cabinet de radiologie,}

A propos de ce lieu particulier qu'est le cabinet de radiologie, il faut rappeler que c'est un lieu marqué par un contexte conflictuel, les médecins radiologistes prenant souvent ombrage de se voir prescrire leurs actes par leur collègues alors qu'ils considèrent qu'ils devraient être destinataires d'un courrier confraternel et décider eux-mêmes de l'examen radiologique. Bref, le radiologue est-il un vrai médecin ${ }^{5}$ ?

La page 40, précisément, à la fois positionne l'espace comme médical et, contradictoirement, donne au patient lui-même des consignes médicales qui normalement devraient être de la responsabilité d'un médecin :

«Certains examens doivent être réalisés de préférence dans la première phase $d u$

cycle chez la femme, etc... ». 

ceux de la luxueuse dépense technologique et ceux de la régulation du marché de l'industrie pharmaceutique? Visiblement les acteurs concernés, faute d'arguments, n'ont pas souscrit à ce nouveau contrat. Il ne s'est pas verbalisé grand chose de ce conflit. Sans doute les partisans assidus de la modernisation y ont-ils vu un exemple de plus de " résistance au changement ", sans trop s'y attarder, pressés de se lancer dans l'aventure de la communication en réseau assistée par l'électronique via le sésame de la carte «Vitale ». On proposera de retenir ce terme de «résistance »: résistance passive, peu concertée mais massive à un changement qui s'avançait masqué, qui n'avait été ni suffisamment analysé ni correctement discuté ni valablement négocié. Un changement qui voulait modifier la construction de la publicité du système de soins sans égard pour l'adossement de cette publicité à des relations de type privé, du client au médecin, du patient à son propre corps, de l'usager-nomade à sa propre liberté de circulation, sans autre considération pour ces relations que quelques dénégations proches de l'aveu involontaire. Un changement dont les promoteurs n'avaient peut-être pas eux-mêmes perçus les enjeux avant que l'échec ne le leur permette. Il faut leur reconnaître que cet échec a été l'occasion d'une expérimentation communicationnelle à grande échelle dont l'analyse des enjeux sociopolitiques et sociosémiotiques du service public a pu beaucoup apprendre. 


\section{BIBLIOGRAPHIE}

Chantraine, O., (1992), « Les écritures professionnelles ou la difficile synthèse de normes communicationnelles hétérogènes » in Études de Communication $\mathrm{n}^{\circ}$ 13, pp. 139-156, Lille.

Chantraine, O., (1992), «Quelques exemples pour l'identification des espaces sociodiscursifs dans les écritures professionnelles » in Études de Communication nº 13, pp. 157-168, Lille.

Chantraine, O., (1997), Approche sociosémiotique d'un espace public fragmenté, Note d'Habilitation à diriger les recherches, Université Paris Nord.

Delcambre, P., (1997), Écritures et communication de travail - Pratiques d'écriture des éducateurs spécialisés, Presses Universitaires du Septentrion, Lille.

Lacoste, M., (à paraître), «Les 'objets' et le travail collectif » in Objets et pratiques de communications organisationnelles : construire des approches scientifiques, Presses Universitaires de Rennes.

Rollet-Echallier, C., (1990), La politique à l'égard de la petite enfance sous la IIIe république, INED/ P.U.F., Paris.

\section{NOTES}

1. En mai 2000 , date où j'écris ce texte, le « trou de la sécu » vient officiellement d'être comblé : la sécurité sociale n'est plus en déficit, principalement renflouée par la reprise de l'activité économique et une relative baisse du taux de chômage. Où l'on voit l'incertitude des liens entre les moyens et les fins. Le carnet de santé est resté inusité et son principal argument officiel a disparu... Il y a même eu des mauvaises langues pour faire observer que ce carnet avait coûté fort cher et donc contribué à la pérennisation du déficit.

2. Nombre d'organisations syndicales de médecins ont ironisé sur le coût de cette diffusion (chiffré à plusieurs centaines de millions de francs dans certains discours) et sous-entendu que cet argent aurait pu être mieux employé... ou économisé.

3. A titre non-obligatoire, le cabinet du chirurgien-dentiste peut être lui aussi concerné.

4. Pour mémoire, la page 48 est prévue pour des notes "personnelles », non préformatées. La personne a donc le loisir d'écrire sur un quarante-huitième de l'espace prévu pour le suivi médical dans son carnet de santé "personnel » et ces notes ne sont pas intégrées au «suivi médical ».

5. P. 5 le carnet soulève la même question à propos des médecins du travail et des médecins des compagnies d'assurance, puisqu'il dit: «Ce carnet de santé ne doit être communiqué qu'aux médecins [...]. Personne d'autre ne peut avoir accès à son contenu, ni les médecins du travail, ni les médecins des compagnies d'assurances, ni votre employeur ». 


\section{RÉSUMÉS}

En 1996, les ministres français chargés de la santé publique lancent le « carnet de santé » dans le but de rationaliser les dépenses de santé. Ce carnet, bien que présenté comme " obligatoire ", resta le plus généralement inusité du fait de la résistance passive et tacite des usagers et des professionnels. L'article propose une analyse sociosémiotique de cet outil de communication. Son échec est celui d'une vaine tentative de modifier les contrats communicationnels d'un espace public professionnel par une innovation autoritaire.

In 1996, the French public health authorities tried to introduce an innovative compulsory medium known as the "health logbook." This document was supposed to be carried by every citizen from one physician to another in order for the professionals to record various information aiming to create a memoir of the patient's health. It was also supposed to be controlled by medical experts in the Social Security administration. This paper focuses on the socio-semiotic specificities of this document, which led it to be widely shunned by the actors despite the fact that its high cost was shared among all citizens. Tacit legitimate resistance led everyone to avoid making use of this logbook, and the authorities finally dropped it. The paper suggests that this failure is related to a desperate attempt to change the rules and conventions of a public professional sphere via authoritarian innovation.

\section{INDEX}

Mots-clés : écriture professionnelle, santé, sécurité sociale, technique de communication, espace public, médecine

Keywords : health card, health, social security, communication technique, public sphere, medecine

\section{AUTEUR}

\section{OLIVIER CHANTRAINE}

Olivier Chantraine est Professeur en Sciences de l'Information et la Communication à l'Université Charles-de-Gaulle - Lille 3. Depuis 1999, il est directeur du GERICO-Lille 3 (Groupement des équipes de recherche interdisciplinaire en communication de l'Université Charles-de-Gaulle - Lille 3). Il y participe à l'équipe « communication organisationnelle ». Ses recherches ont porté sur la pédagogie de l'écriture, les pratiques d'écriture en contexte de travail, la communication publicitaire dans les espaces de la vie ordinaire et l'approche sociosémiotique des espaces publics. 